\title{
Electrodic voltages in the presence of dissolved sulfide: Implications for monitoring natural microbial activity
}

\author{
Lee Slater ${ }^{1}$, Dimitrios Ntarlagiannis ${ }^{1,4}$, Nathan $\mathrm{Yee}^{2}$, Michael O'Brien ${ }^{1}$, \\ Chi Zhang ${ }^{1}$, and Kenneth $\mathrm{H}$. Williams ${ }^{3}$
}

\begin{abstract}
There is growing interest in the development of new monitoring strategies for obtaining spatially extensive data diagnostic of microbial processes occurring in the earth. Open-circuit potentials arising from variable redox conditions in the fluid local-toelectrode surfaces (electrodic potentials) were recorded for a pair of silver-silver chloride electrodes in a column experiment, whereby a natural wetland soil containing a known community of sulfate reducers was continuously fed with a sulfate-rich nutrient medium. Measurements were made between five electrodes equally spaced along the column and a reference electrode placed on the column inflow. The presence of a sulfate reducing microbial population, coupled with observations of decreasing sulfate levels, formation of black precipitate (likely iron sulfide),
\end{abstract}

elevated solid phase sulfide, and a characteristic sulfurous smell, suggest microbial-driven sulfate reduction (sulfide generation) in our column. Based on the known sensitivity of a silver electrode to dissolved sulfide concentration, we interpret the electrodic potentials approaching $700 \mathrm{mV}$ recorded in this experiment as an indicator of the bisulfide ( $\mathrm{HS}^{-}$) concentration gradients in the column. The measurement of the spatial and temporal variation in these electrodic potentials provides a simple and rapid method for monitoring patterns of relative $\mathrm{HS}^{-}$concentration that are indicative of the activity of sulfate-reducing bacteria. Our measurements have implications both for the autonomous monitoring of anaerobic microbial processes in the subsurface and the performance of self-potential electrodes, where it is critical to isolate, and perhaps quantify, electrochemical interfaces contributing to observed potentials.

\section{INTRODUCTION}

An expanding volume of literature demonstrates that electric signals result from the geochemical and physical alteration of soils often driven/induced by microbial processes. Observations of high electric conductivities associated with elevated ionic concentrations in pore fluids, as a result of microbial breakdown of organic contaminants (Atekwana et al., 2000; Atekwana et al., 2004), have encouraged new biogeophysical research in recent years. The macroscale electric signatures in porous media resulting from elevated cell concentrations (Ntarlagiannis et al., 2005), cell growth and biofilm formation (Davis et al., 2006), and biomineralization of metal sulfides under anaerobic conditions (Ntarlagiannis et al., 2005; Williams et al., 2005) have all been investigated in controlled laboratory condi- tions using active electric resistivity and induced polarization geophysical measurements.

The self-potential (SP) method has been suggested as a potentially powerful technique in biogeophysics research (Naudet et al., 2003; Naudet et al., 2004; Ntarlagiannis et al., 2007). This work has been largely based on measurements made at Entressen landfill in France that have been interpreted as evidence for the generation of a biogeobattery whereby biofilms at the water table permit electron shuttling between anoxic (below the water table) and oxic (above the water table) regions of the subsurface (Naudet et al., 2003). The proposed mechanism is similar to the classic geobattery model proposed to explain large $S P(\sim 1 \mathrm{~V})$ measurements observed over metal sulfide ore bodies (Sato and Mooney, 1960). Whereas the metallic

Manuscript received by the Editor 18 July 2007; revised manuscript received 21 September 2007; published online 5 February 2008.

${ }^{1}$ Dept. of Earth \& Environmental Sciences, Rutgers University, Newark, New Jersey, U.S.A. E-mail: 1slater@ andromedia.rutgers.edu; d.ntarlagiannis@ qub.ac.uk; chizhang @ pegasus.rutgers.edu.

${ }_{3}^{2}$ Dept. of Environmental Sciences, Rutgers University, New Brunswick, New Jersey, U.S.A. E-mail: nyee @ envsci.rutgers.edu.

${ }^{3}$ Lawrence Berkeley National Lab, Berkeley, California, U.S.A. E-mail: khwilliams@ @lbl.gov.

${ }^{4}$ Now at School of Planning, Architecture and Civil Engineering, Queen's University Belfast, Belfast, U. K.

(C) 2008 Society of Exploration Geophysicists. All rights reserved. 
mineral body is proposed to provide the electron transport in the traditional geobattery, the biofilms are assumed to perform this function in the biogeobattery (Ntarlagiannis et al., 2007). Microbial electron transfer facilitates respiration and, in many instances, is the dominant mechanism driving organic matter degradation and nutrient cycling processes (Lovley et al., 2004). Microbes have also recently been shown to utilize a variety of conductive appendages to facilitate electron transport to exogenous materials, such as electrodes and metal oxides (Reguera et al., 2005; Gorby et al., 2006). An important requirement of SP measurements, whereby the intent is to detect the electric current induced by remote current sources in the earth, is that no electrodic reactions occur at the electrode surface.

Metallic electrodes are commonly used for electrochemical applications to measure geochemical parameters (e.g., $\mathrm{E}_{\mathrm{h}} \mathrm{pH}$ ), as well as specific chemical concentrations (e.g., $\mathrm{Br}^{-}$using a bromide specific electrode). A common target compound is sulfide, which can be accurately monitored using Ag-AgS electrodes (Berner, 1963; Whitfield, 1971). The basis for such measurements is a galvanic-cell (GC) effect that relies on the known reactivity between the target compound and the electrode used, with the resulting electrodic potential being measured relative to an electrode with a fixed potential. More complex reactive electrode-based techniques, such as voltammetry, have long been utilized to determine chemical speciation associated with sulfide redox chemistry in natural environments, such as the deep ocean (Luther et al., 2001). Although GC and voltammetry methods are fundamentally different (passive versus active), both are based on the principle of electrode surface reactivity with the dissolved substances. Such electrodic reactions are also the basis of the benthic microbial fuel cell (Finkelstein et al., 2006), whereby electric power can be generated in marine sediments by connecting an anode buried in the anaerobic subsurface to a cathode in the overlying oxic water.

Williams et al. (2007) showed that a pair of $\mathrm{Ag}-\mathrm{AgCl}$ electrodes, as sometimes used in the construction of an SP electrode, records a

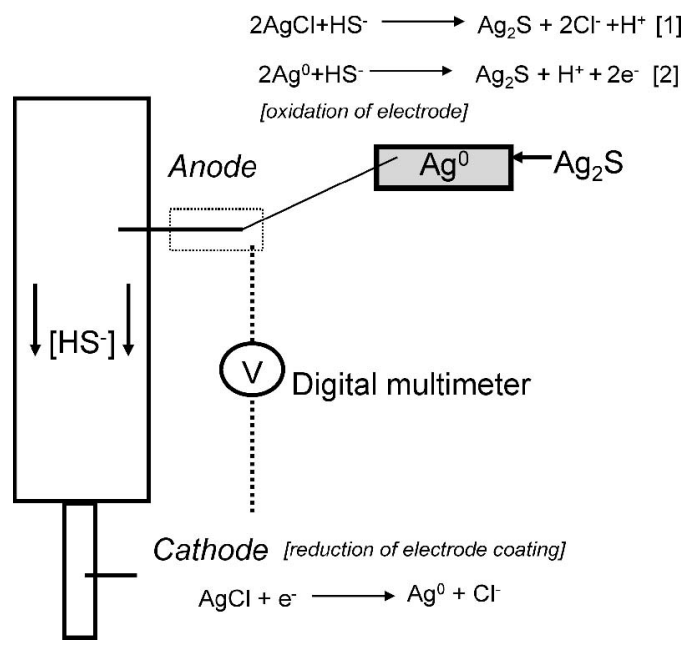

Figure 1. Summary of the galvanic cell reactions for a $\mathrm{Ag}-\mathrm{AgCl}$ electrode pair in the presence of a $\mathrm{HS}^{-}$concentration gradient based on Williams et al. (2007). In the column, oxidation of the electrode occurs as a result of a reaction with bisulfide ions produced following microbial sulfate reduction (the anode becomes an $\mathrm{Ag} / \mathrm{Ag}_{2} \mathrm{~S}$ electrode as per the first reaction in the figure). The cathodic reaction at the reference electrode is a reduction of the $\mathrm{AgCl}$ electrode coating. The $\mathrm{Ag}-\mathrm{AgCl}$ electrode pair acts as a relative bisulfide concentration sensitive redox probe.
GC potential when they straddle a gradient in bisulfide ( $\mathrm{HS}^{-}$) concentration. The basis of this GC is summarized in Figure 1. Oxidation of the $\mathrm{Ag}-\mathrm{AgCl}$ anode results from a half-cell reaction in the presence of elevated $\mathrm{HS}^{-}$concentrations relative to the cathode, whereas reduction of the $\mathrm{AgCl}$ electrode coating occurs at the reference electrode (cathode). The anode effectively becomes an $\mathrm{Ag} / \mathrm{Ag}_{2} \mathrm{~S}$ electrode as per the first reaction in Figure 1. When connected between these electrodes, a voltmeter will record the open circuit potential caused by the differences in $\mathrm{HS}^{-}$concentration at the local fluid-electrode interface between the two electrode locations. This open-circuit potential is a measure of the tendency of a spontaneous reaction between these electrodes to proceed (significant current flow does not occur because of the high input impedance of the voltmeter). The open-circuit potential between the $\mathrm{Ag}-\mathrm{AgCl}$ electrode pair increases with an increase in the $\mathrm{HS}^{-}$concentration difference between the two electrodes (Williams et al., 2007).

Here, we report on temporal changes in the galvanic signature of natural wetland soils associated with changes in redox chemistry and production of $\mathrm{HS}^{-}$mediated by native microbial communities present within the soil using $\mathrm{Ag}-\mathrm{AgCl}$ electrode pairs. We describe measurements made on wetland soils extracted from a freshwater marsh in the Meadowlands of northern New Jersey. These native soils were emplaced in laboratory columns and continually flushed with a nutrient medium to stimulate growth of the sulfate reducing microbial communities under anaerobic conditions. We monitored electrodic potentials on six pairs of electrodes over an $\sim 800$ hour period using a high-input impedance voltmeter.

The purpose of presenting these results is twofold. First, we show how such electrodic signals provide a simple yet diagnostic measure of spatial and temporal variability in natural microbial-induced redox chemistry. The simple electrodes required for the measurement could provide an inexpensive, low maintenance alternative to standard redox probes for the spatiotemporal monitoring of microbial activity using large electrode grids coupled to autonomous geophysical instrumentation. We stress that the measurements described here are a way to sense in situ chemistry by direct contact with the electrode, and are fundamentally different to SP signals arising from the electric field associated with a remote current source. Thus, although of electrochemical origin, these signals are not the SP electrochemical potentials (sometimes called junction potentials) that result from a gradient in ionic concentration giving rise to an electric field parallel to the concentration gradient if cations and anions have different ionic mobilities (Mainueult et al., 2004). Secondly, our results highlight the need for careful consideration of SP electrode design when exploring SP signals associated with processes where electrochemically reactive species are present in the vicinity of the electrode surface.

A fundamental requirement of a conventional SP electrode used to measure potentials associated with remote current sources in the earth is that electrodic reactions involving the electrode are absent. Any electrodic reaction in an SP survey essentially constitutes noise. This requirement is met by using an identical pair of electrodes where the metal (e.g., Ag-AgCl) is in contact with a saturated solution of its own salt (in the porous pot). By maintaining the same (saturating) concentrations at both electrodes, there can be no open circuit potential difference because the local conditions of the electrode-fluid interface are identical. The use of high concentration saturating solutions, combined with long porous pots, minimizes the likelihood of contamination by diffusion of electroactive species (e.g., HS $^{-}$in our case) into the pot toward the metal. SP surveys are 
normally conducted at the oxic earth surface where these requirements are easily met. However, there is growing interest in using SP measurements for long-term monitoring of subsurface processes, for example using electrodes placed down wells (Minsley et al., 2007). In such applications, we feel it important to recognize that such sulfate reducing conditions could potentially promote electrodic reactions if downhole long-term SP monitoring electrodes were poorly designed and vulnerable to contamination by diffusion of bisulfide (such electrodic potentials would be considered noise in an SP survey). In our study, we purposely use $\mathrm{Ag}-\mathrm{AgCl}$ electrodes that should never be used alone as an SP electrode, because we remove the saturating solution so that the potential measured with a voltmeter depends on the surrounding fluid chemistry. However, we show that such electrodic potentials, being very simple to measure, could readily be used to define redox profiles associated with microbial activity in the subsurface.

\section{EXPERIMENTAL METHODS}

Two columns (one active and one control) were dry packed with a mixture (equal concentrations by weight) of wetland soil from the edge of a freshwater wetland in New Jersey, and sterilized sand (100\% silica sand from Ottawa, Ill., 20-30 Mesh). The wetland soil is a soft, brown, saturated peat with an organic matter content of $86.7 \% \pm 0.8 \%$, a moisture content of $77.2 \% \pm 0.9 \%$, and an average Fe content of $1 \%$ (based on 10 undisturbed samples reported in Table 1 of Mansoor and Slater [2007]). These wetland soils are known to contain an indigenous community of sulfate reducing microbes (Desulfovibrio and Desulfotomaculum) (Cai and Hahn, 2002). The control column mixture was autoclaved to kill the indigenous microorganisms present in the wetland soil. All tubing and column components were sterilized by either autoclaving, or with ethanol, to minimize contamination. Both columns were then attached to an open-flow system with an influent nutrient medium to promote microbial growth (Figure 2).

The nutrient medium contained $\mathrm{K}_{2} \mathrm{HPO}_{4}(0.225 \mathrm{~g} / \mathrm{L}), \mathrm{KH}_{2} \mathrm{PO}_{4}$ $(0.225 \mathrm{~g} / \mathrm{L}), \quad \mathrm{NaCl} \quad(0.460 \mathrm{~g} / \mathrm{L}), \quad\left(\mathrm{NH}_{4}\right)_{2} \mathrm{SO}_{4} \quad(0.225 \mathrm{~g} / \mathrm{L})$, $\mathrm{MgSO}_{4} \cdot 7 \mathrm{H}_{2} \mathrm{O}(0.117 \mathrm{~g} / \mathrm{L}), \mathrm{NaHCO}_{3}(0.05 \mathrm{~g} / \mathrm{L}), \mathrm{FeCl}_{3}(0.005$ $\mathrm{g} / \mathrm{L})$, yeast extract $(0.05 \mathrm{~g} / \mathrm{L})$, casamino acids $(0.1 \mathrm{~g} / \mathrm{L})$, acetate $(1.0 \mathrm{~g} / \mathrm{L})$, and Focht trace element solution $(1 \mathrm{~mL} / \mathrm{L})$ (Dungan et al., 2003). The electric conductivity of this medium $\left(\sigma_{\mathrm{w}}\right)$ was $0.099 \pm 0.015 \mathrm{~S} / \mathrm{m}$ throughout the experiment. Measurements on the experimental column consisted of two basic parts: (1) an active flow stage (0-580 hours) where the salts medium was passed through the column at a constant rate of one pore volume/day $(\sim 215$ $\mathrm{ml} /$ day); (2) a zero flow stage (580-800 hours), whereby the flow into the column was stopped by shutting the inflow valve at 580 hours, but potentials still monitored. The control column was terminated at 578 hours as no significant electric signals had been recorded, allowing the column to be used for testing a solid phase extraction technique employed here (Vandevivere and Baveye, 1992). The experiment was performed at a temperature of $25^{\circ} \mathrm{C} \pm 1^{\circ} \mathrm{C}$.

Electric potentials were recorded with a digital multimeter (input impedance $>10 \mathrm{MOhm})$. Complex resistivity $(0.1-1000 \mathrm{~Hz})$ was also recorded, using a dynamic signal analyzer as described by Ntarlagiannis et al. (2005). Voltages were measured using six-point Ag$\mathrm{AgCl}$ electrodes installed in chambers placed $3 \mathrm{~cm}$ apart along the edge of the column (Figure 2). A fine mesh prevented the soil matrix from entering the chambers, but allowed the electrolyte to fill the chambers and establish electrolytic contact between the electrode and the inner part of the column. We followed standard chloriding procedures where $99.999 \%$ pure Ag wires were cleaned with ethanol and then immersed in strong chlorine solution for at least 30 minutes, until a characteristic purple-gray color was observed. Electric potentials were measured between each of these electrodes on the column (E1-E6) relative to a reference electrode (negative terminal of the voltmeter, $E_{\text {ref }}$ in Figure 2a) placed in the tubing of the column inlet. Electric current for complex resistivity measurements was passed through the column using $\mathrm{Ag}-\mathrm{AgCl}$ coils at column ends. Complex resistivity was recorded between three point electrode pairs (E1-E2, E3-E4, and E5-E6). Electric potentials were recorded 2-3 times/day, whereas complex resistivity was measured daily.

Geochemical measurements of $\mathrm{E}_{\mathrm{h}}, \mathrm{pH}$, and fluid conductivity $\left(\sigma_{w}\right)$ of the inflow and outflow were made twice daily throughout the experiment. Following syringe extraction of fluid from inflow and outflow tubes, an $\mathrm{E}_{\mathrm{h}}$ microelectrode was immediately immersed in each fluid to minimize exposure of the fluid sample to atmospheric air. Microprobes were subsequently used to measure $\mathrm{pH}$ and $\sigma_{w}$. Effluent samples were preserved frozen and subsequently analyzed for major anions, such as sulfate, using ion chromatography (IC). Sulfate concentration was used to provide an indirect proxy for sulfide production in the column. Upon completion of the experiment, solid phase samples were extracted from both columns following a procedure for sampling at multiple depths with minimal disturbance (Vandevivere and Baveye, 1992). Solid phase samples were studied using scanning electron microscopy (SEM) imaging with energy dispersive X-ray spectroscopy (EDX) to facilitate elemental analysis.

\section{RESULTS}

Black precipitates were visually observed to form in the active column after $\sim 250$ hours of continuous flow. SEM/EDX suggests

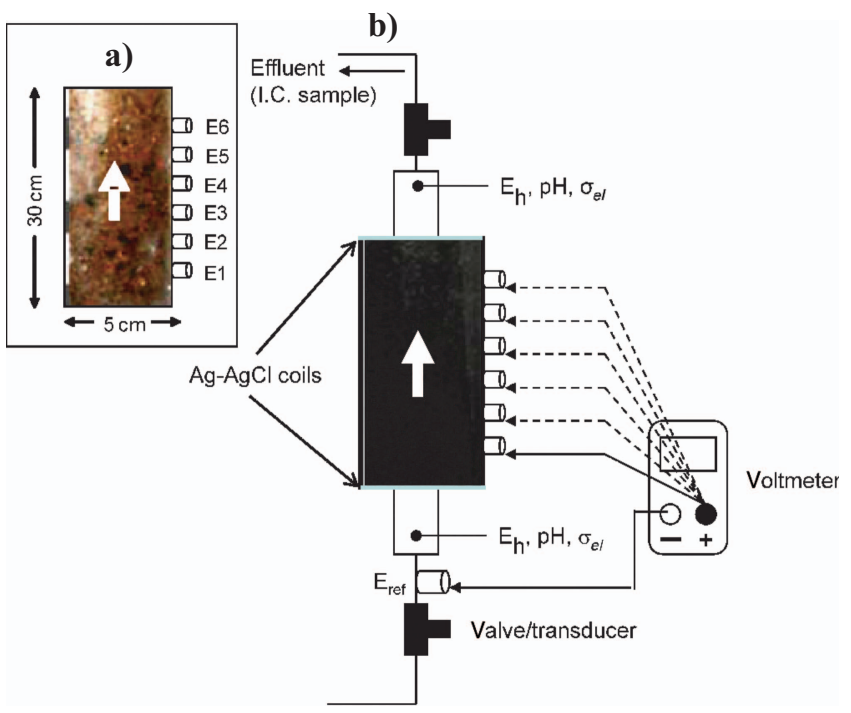

Figure 2. (a) Column dimensions, position of $\mathrm{Ag}-\mathrm{AgCl}$ potential electrodes (E1-E6) and photograph showing color of control column at $t=572$ hours (visually unchanged since start of experiment). (b) Instrumentation showing column flowthrough, GC reference electrode $\left(E_{\text {ref }}\right)$, valve/pressure transducers, and microelectrodes $\left(E_{h}\right.$, $\mathrm{pH}$, and $\left.\sigma_{e l}\right)$. Complex resistivity $(0.1-1000 \mathrm{~Hz})$ was measured with a dynamic signal analyzer between E1-E2, E3-E4, and E5-E6 with current injected between coil electrodes on sample end (instrumentation not shown for simplicity). Here photo shows color of active column at $t=572$ hours 
the precipitant is sulfide in the form of iron sulfide ( $\mathrm{FeS})$ [natural iron concentrations in these soils are high, on the order of 1\%-3\%, Mansoor and Slater (2007)]. No such precipitate was observed for the control column over the duration of the experiment. Precipitation was observed to initiate as isolated sporadic lenses throughout the column length, later coalescing to form distinct precipitant bands in the near vicinity of the original lenses, finally covering the entire active column (Figures $2 b$ and $3 b$ ). Figure $2 a$ is a photograph of the control column at 572 hours of the experiment. The control column did not visibly change color throughout experiment, and the color shown in Figure $3 \mathrm{a}$ is also representative of the color of the active column at the start of experiment. The color of the active column at 572 hours, after extensive precipitant formation, is shown in Figure 2b. Although sulfate inflow concentration analyzed with IC remained constant $(\sim 34 \mathrm{ppm})$ for the duration of the flow through experiment, the outflow concentration declined to virtually zero $(<1 \mathrm{ppm})$ after 300 hours. In contrast, no significant change in sulfate concentration was observed in the control column. The depletion of sulfate in the biologically active system occurred concurrent with the formation of black precipitates within the column. We assume microbial induced sulfate reduction, leading to insoluble sulfide mineral precipitation, was the dominant process in our system because of the initial predominance of sulfate reducers within these soils (Cai and Hahn, 2002). We attribute the nonuniform pattern of precipitation (Figure $3 \mathrm{~b}$ ) to spatial variation in the sulfate reducing microbial population within the column. Biogenic $\mathrm{HS}^{-}$concentrations presumably first increase in areas where the sulfate reducers are most concentrated, and gradually expand to encompass the length of the column. Compared to the control, SEM/EDX analysis identified elevated levels of sulfur in the solid phase of the biologically active column, thereby supporting this interpretation. Furthermore, the characteristic sulfurous smell was readily noted during sampling of effluent. Finally, column electrodes developed a dark black coating indicating the precipitation of $\mathrm{Ag}_{2} \mathrm{~S}$ on the electrode surface as per the expected reaction with $\mathrm{HS}^{-}$(reaction 1 in Figure 1).

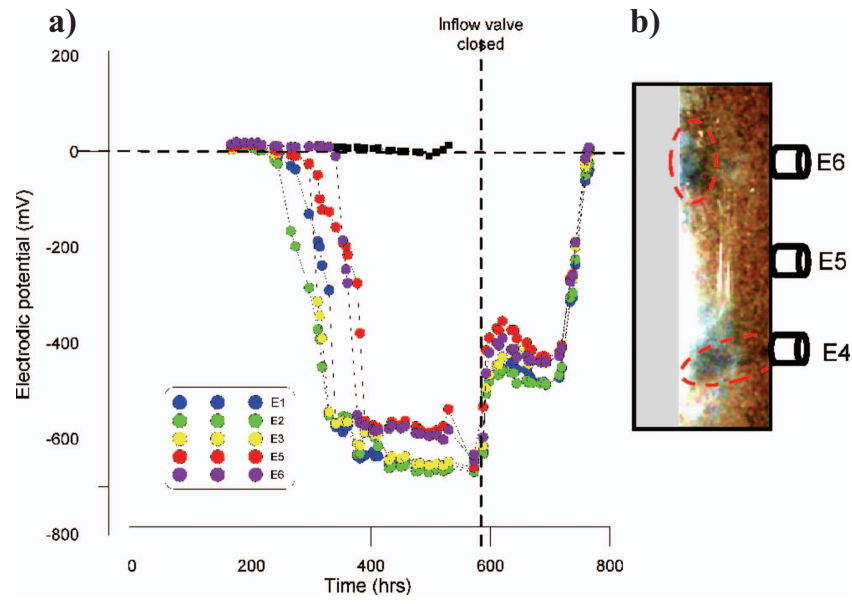

Figure 3. (a) Potential readings during the column experiment as a function of time. Circles denote experiment column locations (lightest shading toward inflow). Open squares denote average of control column readings (maximum standard deviation at any single location in the control column is $3.5 \mathrm{mV}$ ). (b) Close-up of photograph of column showing heterogeneous precipitation (black) during early phase of color change ( $t=300$ hours). Gray shading is cropped area because of excessive glare from the flash.
Figure 3 a shows the potentials recorded at all electrodes on the active column relative to the reference electrode on the inflow. The average of the potentials for E1-E6 on the control column, all relative to the reference electrode, is shown for comparison. Negative potentials (this polarity consistent with the galvanic cell shown in Figure 1) in the active column develop starting at $\sim 250$ hours into the experiment concurrent with the consumption of sulfate detected with IC and observations of visibly dark mineral precipitation. After $\sim 380$ hours, peak negative values $(-550$ to $-680 \mathrm{mV}$ ) were measured for all electrodes, being maintained until closing the inflow valve. Figure 4 shows that there is a general, trend of electrodic potentials occurring at an earlier time, and more negative, toward the inflow, i.e., toward the source of sulfate. However, this trend is not exact as, for example, E4 responds prior to E3. Such variability along the column presumably reflects spatial variability in the HS ${ }^{-}$ concentration driven by the spatially variable microbial community discussed above and visibly evident in the nonuniform precipitant pattern (Figure 3b). Closing the inflow valve of the active column at 580 hours caused a rapid reduction in the electrodic potential with values subsequently stabilizing after $\sim 40$ hours at -390 to -490 $\mathrm{mV}$; these signals were maintained (more negative values generally closer to inflow) until $\sim 720$ hours when the potentials reduced rapidly. By 790 hours, potentials had returned almost to background levels (Figure 3a). Electric potentials in the control column remained steady $(+7 \pm 6 \mathrm{mV})$ for the duration of the experiment. These signals represent the integrated effect of any SP streaming potential, SP electrochemical potential associated with ion diffusion within the column, and any electrodic potentials associated with species other than sulfide in solution. The polarity of the potential on the control column is consistent with a small, streaming self-potential signal resulting from coupling of fluid flow and ionic transport in the electric double layer (e.g., Ishido and Mizutani, 1981).

Complex resistivity measurements revealed no significant change in the electric conductivity $\left(\sigma^{\prime}\right)$ or polarization $\left(\sigma^{\prime \prime}\right)$ coincident with time of precipitate formation. The $\mathrm{E}_{\mathrm{h}}$ recorded at the inflow and outflow fluctuated significantly, probably reflecting problems with

a)

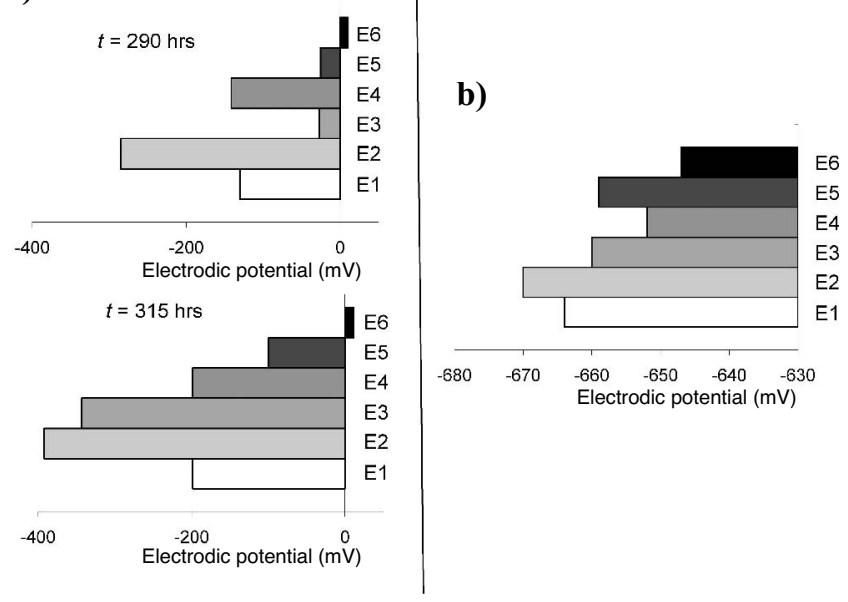

Figure 4. (a) Potentials at two times during the period of rapid electrodic potential change highlighting the nonsmooth distribution along the column between inflow and outflow. (b) Maximum potentials for the entire experiment period observed along the column again displaying a nonuniform distribution between inflow and outflow. 
sampling and exposure to atmospheric air prior to measurement with the microelectrode. However, between $t=220-350$ hours the measurements were relatively stable for the active column, with consistently positive Eh values (50 to $90 \mathrm{mV}$ ) at the inflow and consistently negative $(-70$ to $-145 \mathrm{mV})$ at the outflow.

\section{DISCUSSION}

The signal recorded in the active column during this experiment does not primarily result from a self-potential source mechanism within the column, but instead results from a well-established electrodic reaction between the $\mathrm{Ag}-\mathrm{AgCl}$ electrodes and $\mathrm{HS}^{-}$, in this case, produced as a result of anaerobic microbial activity. The geochemical and visual observations support the microbial production of sulfide by anaerobic microbial sulfate reduction. A potential reaching $-670 \mathrm{mV}$ was observed in the biologically active column, whereas only a small potential $(6-10 \mathrm{mV}$ primarily attributed to a self-potential streaming potential) was detected in the sterile (control) column. It is possible that a self-potential signal associated with the microbial processes in the active column is superimposed on the electrodic potential associated with the HS ${ }^{-}$concentration gradient between the electrodes. We assume that any SP signal is small relative to the electrodic potential because there is no proven SP source mechanism associated with the microbial processes occurring in our columns. Although our nutrient medium contains a mixture of redox-active ions (e.g., sulfate, carbonate), the consistent and small $(+7 \pm 6 \mathrm{mV})$ potentials recorded on the control column, throughout the experiment, prove that these ions do not significantly react with the $\mathrm{Ag}-\mathrm{AgCl}$ electrodes. Thus, we primarily attribute the electrodic signal to a reaction with microbially produced $\mathrm{HS}^{-}$in the active column, accepting that there is a possibility that other redox active ions could contribute about $1 \%-2 \%$ of the measured electrodic potential based on the control column data.

Our results demonstrate that this electrodic measurement itself is a diagnostic tool for rapidly mapping spatial patterns of $\mathrm{HS}^{-}$concentration that could be used to interpret anaerobic microbial activity. Our study of a natural wetland soil is significant because the microbial-driven biogeochemistry of redox processes in wetlands, particularly source-sink cycling of sulfur, remains poorly understood and increasingly relevant as a result of global climate change and modification of near-surface soil chemistry by human inputs (e.g., Alewell et al., 2006). For example, microbial sulfate reduction under anaerobic conditions is coupled to source-sink cycling of iron and heavy metal contaminants in wetland soils. The production of HS ${ }^{-}$ by sulfate reducers is a good indicator of wetland health because it is dramatically reduced by heavy metal contaminants (e.g., Capone et al., 1983). Furthermore, sulfide chemistry in wetlands is considered a critical factor controlling ecosystem diversity.

Although electrodic detection of dissolved sulfide is a wellknown technique, the effect has typically been applied in probes where the sulfide concentration, at a point, is estimated using temperature and $\mathrm{pH}$ data, in addition to the electrodic potential. In our study, we cannot estimate the $\mathrm{HS}^{-}$concentration, but only map the changes in relative $\mathrm{HS}^{-}$concentration along the column, i.e., relative to our reference electrode $\left(\mathrm{E}_{\mathrm{ref}}\right)$. The opportunity thus exists to take the basic concepts of an established electrochemical measurement and apply it in a geophysical-monitoring type of data acquisition, whereby a dense grid of simple $\mathrm{Ag}-\mathrm{AgCl}$ electrodes could be used to map and monitor the spatial variation in $\mathrm{HS}^{-}$concentration relative to a reference electrode (ideally placed at a point of low con- centration, e.g., the earth surface). These electrodes are relatively rugged, inexpensive, simple to construct, and require little maintenance. The procedure could, for example, be applied on a network of electrodes installed in wells to monitor anaerobic microbial activity in the subsurface. Such patterns of electrodic potential, so produced, could provide unique support data to assist the interpretation of more robust field-based methods of $\mathrm{HS}^{-}$detection (e.g., portable gas analyzers), as well as geophysical signals (e.g., resistivity and induced polarization) that may result from anaerobic microbial processes. One obvious application of this measurement, as demonstrated in this laboratory study, is to assist in mapping and monitoring microbial processes in wetlands.

Our experiment has illustrated how such electrodic signals can resolve spatial patterns of $\mathrm{HS}^{-}$related to the spatial distribution of microbial activity. We used wetland soils where the microbial consortia were naturally distributed. The electrodic potentials we mapped develop as the result of the reactivity between the $\mathrm{Ag}$ and $\mathrm{HS}^{-}$when a gradient in $\mathrm{HS}^{-}$concentration is spanned by the two electrodes. The HS $^{-}$concentration depends on both the sulfate availability and the microbial activity. Sulfate availability was highest at the inflow. The nonuniform pattern of precipitation, as emphasized in Figure $3 b$, is therefore probably indicative of the nonuniform microbe population distribution/dynamics within the soil. The drop in potential, after closing the valve, is expected as $\mathrm{HS}^{-}$concentrations reach similar levels along the column and the $\mathrm{HS}^{-}$gradient between the column and reference electrodes decreases. The stabilization of the potential between $t=620-720$ hours possibly suggests that the system temporarily reached an equilibrium state whereby reactants and products temporarily experienced zero net change over time. The equilibrium potential for the cathode-anode pair is $-493 \mathrm{mV}$, close to the values observed during this temporary plateau. After 720 hours the electrodic potential declined to $<50 \mathrm{mV}$ because the $\mathrm{HS}^{-}$gradient between the electrodes induced by the continuous flow disappeared. However, as the reduction potential is highly $\mathrm{pH}\left(\right.$ and $\mathrm{HS}^{-}$) dependent, it is not possible from these results to verify whether the $\sim-500 \mathrm{~m}$ signal indeed results from the equilibrium reduction potential.

Finally, we feel it worthwhile to reflect on the implications of the high sensitivity of $\mathrm{Ag}-\mathrm{AgCl}$ to $\mathrm{HS}^{-}$concentration gradients for the construction of electrodes used for the measurement of self potentials. Similar electrochemical reactions, between $\mathrm{HS}^{-}$and other reactive analytes, exist for other electrodes commonly used in SP surveys (e.g., $\mathrm{Pb}-\mathrm{PbCl}_{2}$ ). As stated earlier, an $\mathrm{SP}$ electrode pair consists of two metal electrodes placed in pots of the same saturating solution so that no potential difference exists between them and no electrodic reactions therefore occur. The possiblity of contamination of the electrode by diffusion of ions into the pot is minimized by constructing pots with a long channel length, fixing the saturating solution with mud/gelling agent, and using a narrow channel in the electrode (Petiau, 2000). Using electrode lengths of $\sim 0.1 \mathrm{~m}$ (and assuming typical diffusivities of $10^{-9}$ to $10^{-10} \mathrm{~m}^{2} / \mathrm{s}$ ), diffusion times are on the order of 20 days to many years (Petiau, 2000). When using SP measurements at depth in boreholes, there is also a possibility of contamination by pressure induced flow into the electrode if it is imperfectly sealed. The strength of the electrodic potentials in the presence of $\mathrm{HS}^{-}$measured in this study demonstrates that even small amounts of contamination could result in significant potentials unrelated to remote SP sources. We therefore recommend that careful attention be given to SP electrode design for monitoring microbial activity in sulfate reducing environments, that the recommendations of 
Petiau (2000) are followed, and that diffusion times are estimated and compared with the expected duration of the monitoring survey. However, if the intent of the geophysical survey is to characterize and monitor redox profiles, we suggest a dual sensor electrode design. In that design, one sensor acts to record the electrodic potential resulting from direct contact between metal electrode and a target compound; whereas, the second sensor is a traditional SP electrode that could be used to detect current sources in the earth resulting from any remote biogeobattery effect if it exists. Our study highlights a need for investigations of electrodic voltages that may arise because of varying degrees of contamination of traditional SP electrodes.

\section{CONCLUSIONS}

We have shown how electrodic potentials that develop on Ag$\mathrm{AgCl}$ electrodes in the presence of dissolved sulfide are indicative of microbial activity within natural soils. These electrodic potentials respond to the microbial-induced changes in the fluid chemistry local to the electrode surface. We assumed that the primary electrodic signal is an open-circuit potential associated with a half-cell reaction involving dissolved sulfide, although it is possible that other redox active ions in solution may also have contributed to the observed signal. The signal we observe is not a self potential resulting from an external current source within the column. We recommend that future biogeophysics-based experiments aimed at the detection of microbial processes with electric methods utilize dual electrode sensors, whereby one sensor is a self-potential electrode (i.e., a metal embedded in a porous pot) and the other is an $\mathrm{Ag}-\mathrm{AgCl}$ electrode in direct contact with the pore-filling fluids, such as employed here. Using such electrodes, it should be possible to improve understanding of anaerobic microbial processes that drive changes in fluid chemistry relative to making self-potential measurements alone.

\section{ACKNOWLEDGMENTS}

This material is based in part upon work supported by the National Science Foundation under grant EAR-0433729. Financial support was also provided from the Meadowlands Environmental Research Institute (MERI) via a 2006 MERI Fellowship award to Lee Slater. Reviews provided by Mathieu Darnet and an anonymous reviewer improved the clarity of this manuscript.

\section{REFERENCES}

Alewell, C., S. Paul, G. Lischeid, K. Kusel, and M. Gehre, 2006, Characterizing the redox status in three different forested wetlands with geochemical data: Environmental Science and Technology, 40, 7609-7615.

Atekwana, E. A., E. Atekwana, F. D. Legall, and R. V. Krishnamurthy, 2004 Field evidence for geophysical detection of subsurface zones of enhanced microbial activity: Geophysical Research Letters, 31, L23503.

Atekwana, E., W. A. Sauck, and D. D. Werkema, 2000, Investigations of geoelectric signatures at a hydrocarbon contaminated site: Journal of Applied Geophysics, 44, 167-180.

Berner, R. A., 1963, Electrode studies of hydrogen sulfide in marine sediments: Geochemica et Cosmochimica Acta, 27, 563-575.

Cai, H., and D. Hahn, 2002, Assessing microbial indicators for heavy metal contamination using automated image analysis: Meadowlands Environment Research Institute (MERI), 1-9.
Capone, D. G., D. D. Reese, and R. P. Kiene, 1983, Effects of metals on methanogenesis, sulfate reduction, carbon dioxide evaluation, and microbial biomass in anoxic salt marsh sediments: Applied and Environmental Microbiology, 45, 1586-1591.

Davis, C. A., E. Atekwana, E. Atekwana, L. D. Slater, S. Rossbach, and M. R Mormile, 2006, Microbial growth and biofilm formation in geologic media is detected with complex conductivity measurements: Geophysical Research Letters, 33, L18403.

Dungan, R. S., S. R. Yates, and W. T. Frankenberger Jr., 2003, Transformations of selenate and selenite by Stenotrophomonas maltophilia isolated from a seleniferous agricultural drainage pond sediment: Environmental Microbiology, 5, 287-295.

Finkelstein, D. A., L. M. Tender, and J. G. Zeikus, 2006, Effect of electrode potential on electrode-reducing bacteria: Environmental Science and Technology, 40, 6990-6995.

Gorby, Y. A., S. Yanina, J. S. McLean, K. M. Rosso, D. Moyles, A. Dohnalkova, T. J. Beveridge, I. S. Chang, B. H. Kim, K. S. Kim, D. E. Culley, S. B. Reed, M. F. Romine, D. A. Saffarini, E. A. Hill, L. Shi, D. A. Elias, D. W. Kennedy, G. Pinchuk, K. Watanabe, S. I. Ishii, B. Logan, K. H. Nealson, and J. K. Fredrickson, 2006, electricly conductive bacteria nanowires produced by Shewanella oneidensis strain MR-1 and other microorganisms: Proceedings of the National Academy of Sciences, 103, 1358-11363.

Ishido, T., and H. Mizutani, 1981, Experimental and theoretical basis of electrokinetic phenomena in rock-water systems and its application to geophysics: Journal of Geophysical Research, 86, 1763-1774.

Lovley, D. R., D. E. Holmes, and K. P. Nevin, 2004, Dissimilatory Fe(III) and Mn(IV) reduction: Advances in Microbial Physiology, 49, 219-286.

Luther, G. W. I., T. F. Rozan, M. Taillefert, D. B. Nuzzio, C. Di Meo, T. M. Shank, R. A. Lutz, and S. Craig Cary, 2001, Chemical speciation drives hydrothermal vent ecology: Nature, 410, 813-816.

Maineult, A., Y. Bernabé, and P. Ackerer, 2004, electric response of flow, diffusion, and advection in a laboratory sand box: Vadose Zone Journal 3, 1180-1192.

Mansoor, N., and L. Slater, 2007, On the relationship between iron concentration and induced polarization in marsh soils: Geophysics, 72, no. 1, A1A5.

Minsley, B., J. Sogade, and F. D. Morgan, 2007, Three-dimensional self-potential inversion for subsurface DNAPL contaminant detection at the Savannah River Site, South Carolina: Water Resources Research, 43, W04429.

Naudet, V., A. Revil, J. Y. Bottero, and P. Bégassat, 2003, Relationship between self-potential (SP) signals and redox conditions in contaminated groundwater: Geophysical Research Letters, 30, 2091

Naudet, V., A. Revil, E. Rizzo, J. Y. Bottero, and P. Begassat, 2004, Groundwater redox conditions and conductivity in a contaminated plume from geoelectric investigations: Hydrology and Earth System Sciences, 8, $8-22$

Ntarlagiannis, D., E. A. Atekwana, R. A. Hill, and Y. Gorby, 2007, Microbial nanowires, Is the subsurface "hardwired"?: Geophysical Research Letters, 34, L17305

Ntarlagiannis, D., K. H. Williams, L. Slater, and S. Hubbard, 2005, The low frequency electric response to microbially induced sulfide precipitation Journal of Geophysical Research, 110, G02009.

Ntarlagiannis, D., N. Yee, and L. Slater, 2005, On the low-frequency electric polarization of bacterial cells in sands: Geophysical Research Letters, 32 L24402.

Petiau, G., 2000, Second generation of lead-lead chloride electrodes for geophysical applications: Pure and Applied Geophysics, 157, 357-382.

Reguera, G., K. D. McCarthy, T. Mehta, J. S. Nicoll, M. T. Tuominen, and D. R. Lovley, 2005, Extracellular electron transfer via microbial nanowires: Nature, 425, 1098-1101.

Sato, M., and H. M. Mooney, 1960, The electrochemical mechanism of sulfide self-potentials: Geophysics, 25, 226-249.

Vandevivere, P., and P. Baveye, 1992, Sampling method for the observation of microorganisms in unconsolidated porous media via scanning electron microscopy: Soil Science, 153, 482-485.

Whitfield, M., 1971, A compact potentiometric sensor of novel design: In situ determination of $\mathrm{pH}, \mathrm{pS}^{2-}$ and Eh: Limnology and Oceanography, 16, 829-837.

Williams, K. H., S. S. Hubbard, and J. F. Banfield, 2007, Galvanic interpretation of self-potential signals associated with microbial sulfate-reduction Journal of Geophysical Research, 112, G03019.

Williams, K. H., D. Ntarlagiannis, L. Slater, A. Dohnalkova, S. S. Hubbard, and J. F. Banfield, 2005, Geophysical imaging of stimulated microbial biomineralization: Environmental Science and Technology, 39, 75927600 . 